\title{
Oxytocin-Gly-Lys-Arg stimulates cardiomyogenesis by targeting cardiac side population cells
}

\author{
Bogdan A Danalache', Calvin $\mathrm{Yu}^{1}$, Jolanta Gutkowska ${ }^{1,2}$ and Marek Jankowski ${ }^{1,2}$ \\ ${ }^{1}$ Research Centre, Centre Hospitalier de I'Université de Montréal (CRCHUM), Montréal, Québec, Canada \\ ${ }^{2}$ Department of Medicine, Université de Montréal, Montreal, Quebec, Canada
}

Correspondence should be addressed to M Jankowski Email marek.jankowski@ umontreal.ca

\begin{abstract}
The functional oxytocin (OT) system is expressed in the human and rodent hearts. OT stimulates differentiation of cardiac stem cells into contracting cardiomyocytes (CM). In this study, we investigated OT receptors (OTR) expressed in the cells of cardiac side population (SP) and the abilities of these cells to differentiate into CM in response to the treatment with OT-Gly-Lys-Arg (OT-GKR), a dominant and biologically active form of OT, in the fetal rodent heart. Immunocytochemistry of whole rat embryo at mid gestation (E11) revealed parallel staining in the heart of OTR and the ATP-binding cassette sub-family G member 2 (brcp1) antigen the marker of the SP phenotype. Using flow cytometry, the SP cells were selected from the newborn CM stained with Höechst 33342: $5.32 \% \pm 0.06 \%$ of SP and $15.2 \% \pm 1.10$ of main population expressed OTR on the cell surface. The OTR was detected in CD29 (6.6\%) and then in CD31 (4.7\%) but less frequently in CD45 (0.7\%) positive SP cell subpopulations. Specifically, the phenotype of SP CD31 - cell, but not SP CD31+ cells, proliferates in the presence of OT-GKR and develops large cell aggregates. Then, OT-GKR treatment induced the apparition of beating cell colonies after 11 days $(10 \pm 2.78 \%)$, which increased until day $16(52 \pm 1.21 \%)$. The cells in contractile colonies expressed the markers of a CM phenotype, such as troponin, cardiac myosin light chain-2, and actinin. Finally, SP cells stimulated by OT-GKR induced endothelial phenotype. These results suggest that the C-terminally extended OT molecule stimulates cardiac differentiation of SP CD31 - cells and is involved in heart growth.
\end{abstract}

\author{
Key Words \\ - oxytocin elongated form \\ - embryo \\ - cardiac side population \\ - oxytocin receptor \\ - cardiac differentiation
}

\section{Introduction}

Oxytocin (OT) originally recognized as a pregnancy hormone is produced primarily in paraventricular and supraoptic nuclei of the hypothalamus. OT is also synthesized in the cardiovascular system (Jankowski et al. 1998, Oyama et al. 2007), where it acts as a potential cardiomyogen and as a cardioprotective factor mediating anti-inflammatory and anti-oxidative actions, regulating cardiac contractility, heart rate, and stimulating glucose uptake in the cardiac cells (Gutkowska \& Jankowski 2012). Furthermore, OT treatment attenuates detrimental effects of myocardial infarction (Jankowski et al. 2010) and activates signals that prevent the death of cardiomyocyte (CM) exerted by ischemia/ reperfusion injury in vivo (Kobayashi et al. 2009) and in isolated perfused heart (Ondrejcakova et al. 2009, Anvari et al. 2012).
Journal of Endocrinology (2014) 220, 277-289 
The expression levels of OT are higher in developing than in adult hearts suggesting that OT may be involved in CM differentiation during heart maturation (Jankowski et al. 2004). In addition, OT, and more prominently, its precursor, extended form OT-Gly-Lys-Arg (OT-GKR), has been detected by immunocytochemistry in the heart of mouse embryo and in the rat fetal heart extracts by reverse-phase HPLC and RIA (Danalache et al. 2010). In the major site of OT production, the hypothalamus, the translation of Ot (Oxt) mRNA generates a precursor peptide containing both OT and neurophysin I (Altstein \& Gainer 1988). The tripeptide Gly-Lys-Arg separates the nonapeptide OT from neurophysin I. In the hypothalamus, posttranslational processing of this peptide involves the cleavage of OT-GKR from neurophysin I. Then Arg and Lys are cleaved by carboxypeptidase B to yield OT-Gly, which is modified by C-terminal $\alpha$-amidating enzyme to mature OT nonapeptide (Altstein \& Gainer 1988). The ability to process the initial translation product through to mature OT is developmentally regulated and increases during late fetal and early neonatal development (Mitchell et al. 1998). In fetal sheep plasma, the concentrations of extended forms of OT are 35-fold higher than that of OT (Morris et al. 1992). Similarly, in human umbilical plasma, a higher concentration of OTextended forms than OT was reported (Mueller-Heubach et al. 1995). Molecular analysis by computerized software demonstrated that OT-GKR and OT have both similar and different docking sites inside the OT receptor (OTR) and receptors of other neurophyseal hormone, arginine vasopressin (AVP; Danalache et al. 2010). This suggested unique and specific OT-GKR effect on the cells. Indeed, experiments ex vivo on embryonic stem cell line D3 (Gassanov et al. 2008) and embryonic carcinoma P19 cells demonstrated that OT-GKR (Danalache et al. 2010) had increased potential to differentiate cells into CM in comparison with amidated-OT molecule.

Adult stem cells are thought to be tissue specific and only able to differentiate into progeny cells of their tissues of origin. Stem cells from bone marrow, muscles, and other tissues can be identified by the 'side population' (SP) phenotype. The SP phenotype is a manifestation of primitive cells' ability to efficiently efflux the fluorescent DNA-staining dye Höechst 33342 and can be used as the basis by which to isolate these cells using flow cytometry. This effect is mediated in part by the ATP-binding cassette sub-family G member 2 (ABCG2) transporters, also termed as breast cancer resistance protein 1 , and recognized as a molecular determinant of the SP phenotype. The SP cells are separated from the main population (MP) when analyzed by dual wavelength flow cytometry cell sorting. In the neonatal mice heart, the SP cells compose $3.5 \%$ of CMs, which is markedly higher than the range of $0.01-1 \%$ that was observed in other organs, including blood, skeletal muscle, and brain (Liang et al. 2010). The SP cell population is specified early during development and presumably contributes to cardiac organogenesis in the embryo (Martin et al. 2004). Because the fetal heart primarily oxidizes glucose and lactate to generate ATP (Fisher et al. 1980), it is important that OT-GKR evokes more potently than OT, the glucose uptake in SP cells (Florian et al. 2010). We hypothesized that OT-GKR can potentially serve as a natural cardiac regulator and cardiomyogen during fetal growth and shortly after birth. Therefore, in this study, we investigated whether the OT-GKR peptide has the potential to induce cardiac differentiation in somatic stem cells of the heart, as these cells have been shown to respond in such a way to OT (Matsuura et al. 2004, Oyama et al. 2007).

\section{Materials and methods}

\section{Immunocytochemistry and microscopic analysis of rat embryo}

For immunohistochemical staining, rat embryos (embryonic day 11) were fixed with $4 \%$ formaldehyde and $0.1 \%$ picric acid in $0.1 \mathrm{M}$ PBS ( $\mathrm{pH} 7.4$ ), embedded in paraffin. About $5-\mu \mathrm{m}$ sections were cut longitudinally and mounted on polylysine-treated slides (Cat. No. P-4981, Esco, Erie Scientific Co., Portsmouth, NH, USA). The antigens were detected by indirect binding with primary specific antibodies and HRP-conjugated secondary antibodies (1:100) with Histostain Plus Rabbit Primary 3,3'-diaminobenzidine substrate (Cat. No. 85-9243, Zymed Laboratories, San Francisco, CA, USA, zymed.com). The primary antibodies (1:30) obtained from Santa Cruz Biotechnology were goat polyclonal anti-OTR (Cat. No. sc-8102), goat polyclonal anti-ABCG2 (Cat. No. sc-25156), and goat polyclonal anti-Nkx-2.5 (Cat. No. sc-8697). The secondary antibodies were donkey anti-goat IgG-HRP (Cat. No. sc-2020, Santa Cruz). Normal goat IgG antibodies (1:30, Cat. No. sc-2028 Santa Cruz) were used as an isotype control. Control also included staining with OTR-specific antibody and pre-absorbed with OTR-neutralizing peptide (Oxytocin-R (C-20) P, Santa Cruz Biotechnology). For pre-adsorption, $2.5 \mathrm{~g}$ neutralizing peptide has been added to $1 \mathrm{ml}$ working dilution of the antiserum and kept overnight at $4{ }^{\circ} \mathrm{C}$. The staining with pre-adsorbed antibodies was negative

Published by Bioscientifica Ltd 
in uterus section as well as in rat fetal sections employed in this study.

Morphology was examined under a Model IX51 inverted microscope (Olympus, Tokyo, Japan) and the micrographs were taken with QICAM-IR Fast 1394 Digital CCD camera. Panoramic, cross-sectional, digital images of stained whole embryos were prepared with Image J, Fiji software (Schindelin et al. 2012) (National Institutes of Health, Bethesda, MD, USA, http://rsbweb.nih.gov/ij/) and Adobe Photoshop CS software (Adobe Systems, Inc.).

\section{Cell culture and isolation of SP by fluorescence-activated cell sorting}

All experiments conformed to the guidelines of the Canadian Council on Animal Care and were approved by the Animal Care Committee of the Centre Hospitalier de l'Université de Montréal. One to four-day-old-neonatal Sprague-Dawley rats (Charles River, St-Constant, Quebec, Canada) were killed and the CMs were prepared using the Neonatal CM Isolation System (Cat. No. LK003300; Worthington, Lakewood, NJ, USA) as already reported (Florian et al. 2010).

The cells were prepared from 21 to ensure heterogeneity of the cell population and suspended in DMEM-low-glucose medium followed by four cycles of plating in 150-mm Petri dishes for $30 \mathrm{~min}$, to selectively remove non-myocytes. The purity of these cells was assessed by fluorescence staining with antibodies specific for Troponin-T (Florian et al. 2010). The cells were stained by Höechst 33342 dye (bisBenzimide H 33342 trihydrochloride, Cat. No. B2261, Sigma) at a concentration of $1 \mu \mathrm{g} / \mathrm{ml}$ and at $37^{\circ} \mathrm{C}$ for $60 \mathrm{~min}$ (Oyama et al. 2007). After centrifugation the cells resuspended in DMEM were kept on ice until fluorescence-activated cell sorting (FACS) separation (Dako Cytomation MoFlo, BD Aria; Mississauga, Ontario, Canada). SP was defined by low fluorescence emitted by the cells, due to the removal of Höechst dye by the ATP-binding cassette transporter, ABCG2. The activity of this protein was inhibited by $50 \mu \mathrm{M}$ verapamil (Cat. No. 381195-1G, Sigma). Following Höechst staining, the cells were stained for the expression of cell surface proteins. For analysis of OTR in living cells, we used an OTR-specific antibody raised against a peptide sequence mapped near the N-terminus of OTR, anti-OTR (Cat. No. sc-8103, Santa Cruz Biotechnology), followed by donkey anti-goat IgG-FITC or donkey Phycoerythrin (PE)-labeled anti-goat IgG. Control included staining with OTR-specific antibody pre-absorbed with OTR-neutralizing peptide (the Oxytocin-R (N-19) sc-8103, Santa Cruz Biotechnology). The FACS-selected, Höechst-stained cells, were incubated with antibodies specific for OTR plus antibody specific for one of the three cell surface proteins, CD31, CD45, and CD29. The conjugates (BD Biosciences, Mississauga, ON, Canada, www.bdbiosciences.ca) included hamster anti-rat monoclonal CD29-FITC (clone Ha2/5, Cat. No. 555005), mouse anti-rat monoclonal CD31 PE (clone TLD-3A12, Cat. No. 555027), and mouse anti-rat monoclonal CD45-PE (clone OX-1, Cat. No. 551402). The cells were analyzed by LSR1 cytometer (Beckton-Dickinson) using software from University of Turku and Åbo Akademi University (www.flowingsoftware.com), Dako Cytomation MoFlo, BD Aria, and WinMDI 2.8. Software from Purdue University.

\section{ABCG2 cell magnetic separation}

Alternatively to isolation of SP cells from the rat heart, the cell phenotype of ABCG2 + CD31 - was separated using flow cytometry and magnetic beads. At first, flow cytometry with mouse anti-rat CD31 antibody was used to separate the CM-enriched cell fraction into CD31- and CD31+ phenotypes. Then, ten million of CD31- cells were centrifuged at $900 \boldsymbol{g}$ for $5 \mathrm{~min}$, resuspended in $100 \mu \mathrm{l}$ MACS buffer, stained with $10 \mu \mathrm{l}$ of the first ABCG2 antibody D-20 (Cat. No. sc-25156) goat polyclonal IgG, from Santa Cruz Biotechnology, and incubated on ice for $1 \mathrm{~h}$. The cells were washed and centrifuged twice with $20 \mathrm{ml}$ buffer to remove unbound primary antibody. Then, the cells were resuspended in $100 \mu \mathrm{l}$ buffer with $10 \mu \mathrm{l}$ secondary R-PEconjugated antibody AffiniPure $\mathrm{F}\left(\mathrm{ab}^{\prime}\right) 2$ fragment rabbit anti-goat IgG (Cat. No. 705-116-147) from Jackson ImmunoResearch Laboratories (West Grove, PA, USA) and incubated on ice in the dark for $14 \mathrm{~min}$. The cells were then washed with $20 \mathrm{ml}$ buffer and spun twice. The cells were resuspended in $1000 \mu \mathrm{l}$ buffer with $200 \mu \mathrm{l}$ anti-PE micro beads (Cat. No. 120-000-294 from Macs Milteny Biotech, Inc., Auburn, CA, USA) and incubated for $15 \mathrm{~min}$ in the dark on ice. Then the cells were filtered with Macs PreSeparation Filter $30 \mu \mathrm{m}$ (Cat. No. 5100712066) and separated magnetically by placing in an activated Macs LS column (Cat. No. 120-000-475) in the magnetic field of Macs device (Cat. No. 130-042-302). The column was washed three times with $2.5 \mathrm{ml}$ Macs running buffer (BSA EDTA PBS 0.09\% azide pH 7, 2 Cat. No. 130-091-221) and the effluent was collected as ABCG2-negative-unlabeled cell fractions and kept on ice. The ABCG2-positive cells retained magnetically were collected from the column in $5 \mathrm{ml}$ buffer and immediately flushed by the plunger supplied with the column. They were cultured for 16 days. Control ABCG2positive cells did not show any signs of beating at the end

Published by Bioscientifica Ltd 
of this period (Supplementary Table 1, see section on supplementary data given at the end of this article).

\section{3-(4,5-dimethylthiazol-2-yl)-2,5-diphenyltetrazolium bromide assay}

To assess the viability of cells, the 3-(4,5-dimethylthiazol2-yl)-2,5-diphenyltetrazolium bromide (MTT) assay was carried out according to the instructions provided by the manufacturer (Cat. No. G3580, www.promega.com). The absorbance of colored solution was quantified at $490 \mathrm{~nm}$ wavelength using Biotek Synergy 4 microplate reader (BioTek Instruments, Winooski's, VT, USA). The absorbance values were expressed as \% over the control.

\section{Cardiac differentiation and aggregate formation}

The cardiomyogenic differentiation of SP cells was carried out according to already published methodology (Danalache et al. 2010). Briefly, the cells were suspended in DMEM-low-glucose with L-glutamine (Cat. No. 11885084) containing $100 \mathrm{U} / \mathrm{ml}$ penicillin-G, $100 \mu \mathrm{g} / \mathrm{ml}$ streptomycin sulfate, $0.25 \mu \mathrm{g} / \mathrm{ml}$ amphotericin $\mathrm{B}$, and $10 \%$ fetal bovine serum. About 1000 cells in $25 \mu \mathrm{l}$ were plated in hanging drops, on the lid of $10-\mathrm{cm}$ bacteriological grade Petri dishes and incubated at $37^{\circ} \mathrm{C}$ for 2 days in the absence (control) or the presence of $10^{-7} \mathrm{M}$ OT-GKR $\left(\mathrm{Gly}^{10}{ }^{1}, \operatorname{Lys}^{11}, \operatorname{Arg}^{12}, 43232 \_1\right)$. The peptide was synthesized by GenScript Corp. (Piscataway, NJ, USA) and the concentration was validated before this study. At day 2 of aggregation, the culture medium was replenished; aggregates formed in drops were transferred to bacterial dishes coated with poly 2-hydroxyethyl methacrylate (Cat. No. P3932, Sigma-Aldrich). Aggregates were kept for 3 more days in suspension in the absence or presence of $10^{-7} \mathrm{M}$ OT-GKR.

The viable cells in aggregates were stained for $40 \mathrm{~min}$ in the solution of $1.5 \mu \mathrm{g} / \mathrm{ml}$ acridine orange and $10 \mu \mathrm{g} / \mathrm{ml}$ propidium. The viable cells emitted green fluorescence under dark field fluorescence microscopy, whereas nonviable cells were red after excitation at $470 \mathrm{~nm}$ and emission fluorescence at $515 \mathrm{~nm}$.

For differentiation, the inducer was present over the 5 days of aggregation, with a new replacement after day 2 . At day 5, eight aggregates/well were plated to 24-well tissue culture grade vessels and cultured in complete medium in the absence of OT-GKR. The appearance of contracting cell colonies was noted at days $6-18$ of the entire differentiation protocol under microscopy and recalculated from video recordings.
For immunofluorescence, the cells grown in Lab-Tek plates (Cat. No. 177437, Nalge Nunc International, Rochester, NY, USA) coated with $0.01 \%$ poly-L-lysine (Cat. No. P4832, Sigma) were fixed in a solution containing $4 \%$ paraformaldehyde in 0.1 M PBS, pH 7.4, for $10 \mathrm{~min}$, washed in PBS, and permeabilized for $10 \mathrm{~min}$ with $0.4 \%$ Triton $\mathrm{X}-100$. For staining of ventricular myosin light chain-2, the goat polyclonal antibody (1:100) (myosin light chain-2 ventricular (MLC2V), C17, Cat. No. sc-34490, Santa Cruz Biotechnology) and then donkey anti-goat IgG antibody conjugated to Alexa Fluor 488 were employed (1:200, Cat. No. A11055, Invitrogen, Life Technologies). For detection of sarcomeric $\alpha$-actinin, the monoclonal mouse antibody (1:800, clone EA-53, Cat. No. 096K4774, Sigma) and goat anti-mouse IgG conjugated to Alexa Fluor 568 (Cat. No. A11004, Invitrogen) were used. Nestin rabbit polyclonal antibody (1:300, Cat. No. Ab5968, Abcam, Inc., Cambridge, MA, USA) was used for the expression of undifferentiated cells using donkey anti-rabbit IgG secondary antibody conjugated to green fluorophore Alexa Fluor 488 (Cat. No. A21206, Invitrogen).

\section{Matrigel cell culture of CD31 - SP cells}

The cells were seeded at 60000 cells per well into a 24-well plates coated with diluted BD Matrigel (Basement Membrane Matrix; Becton Drive, Franklin Lakes, NJ, USA). The OT-GKR was added at $10^{-7} \mathrm{M}$ concentration. The medium was changed on the 1 st day, and after it was replaced every 3 days. Each time the medium was supplemented with the fresh portion of OT-GKR. Only short strands were formed in non-induced SP cells after 14 days. An extensive network of cords and tube-like structures can be seen on Matrigel by OT-GKR-induced CD31 - SP cells after 4 days. At this time, cells were analyzed in contrast phase, fixed in Tissuefix solution (Laboratory Gilles Chaput, Inc., Montreal, Quebec, Canada), and stained with rabbit polyclonal antibodies, specific for von Willebrand factor (vWF, 1:100, Abcam, Cat. No. ab6994).

\section{Statistical analysis}

The results obtained from at least three independent cell series were expressed as mean \pm s.E.M. Comparisons of cell aggregate diameters and numbers of beating cell colonies between groups of differently treated cells were evaluated by one-way ANOVA, followed by Newman-Keuls multiple comparison test with the PRISM GraphPad Software (La Jolla, CA, USA). Statistical significance was taken as $P<0.05$.

Published by Bioscientifica Ltd. 


\section{Results}

\section{Identification of SP cells and cells positive for OTR in the rat embryo}

Immunocytochemistry of whole rat embryos was carried out in mid-gestational age E11 during early organogenesis associated with progressive septation of the atria and ventricles and before coronary vasculogenesis, which first occurs in gestation day 13 (E13) (Tomanek et al. 1996). The heart was localized in the embryonic sections by specific staining of the NK2 cardiac-specific homeo box 5 (NKX2-5) protein (Fig. 1A). Because NKX2-5 is recognized as the earliest known marker of cardiac specification and cardiac lineage (Olson 2006), the parallel staining for OTR (Fig. 1B), and the ABCG2 antigen, the marker of SP phenotype (Fig. 1C), indicated the presence of OTR- and ABCG2-positive cells in the heart area of embryo. As demonstrated in Fig. 1, OTR is ubiquitously expressed in the heart and is not restricted to ABCG2positive cells. No staining with isotype control antibodies was seen in control sections (Fig. 1D and D1). Although the intensive staining of all three proteins was clearly visible in the embryonic heart, the NKX2-5 (Fig. 1A1) and
OTR (Fig. 1B1) were predominantly expressed in the ventricular cells whereas ABCG2 protein signal (Fig. 1C1) was equally intense in the atrium as in the ventricles.

\section{Characteristics of SP cells from the newborn rat heart}

Fluorescent sorting analysis of cells isolated in CM fraction from rat newborn heart revealed two cell populations termed as MP, which included mature CMs, and SP cells (Fig. 2A). These experiments revealed $4.29 \pm 0.83 \%$ of live SP cells detected in the region that characterizes SP population from neonatal rat hearts (shown in R3 window, propidium iodide negative and debris were excluded) in comparison with $74.4 \pm 2.60 \%$ cells detected in MP. Most of the cells identified as a SP population (90\%) were eliminated when verapamil blocked the efflux of the Höechst stain (Fig. 2B). Next, we investigated the presence of specific OTR on the cell surface. Comparison of total cells selected by flow cytometry (Fig. 2C) and the subpopulation of cells separated by OTR antibody (Fig. 2D) demonstrated that $15.2 \pm 0.6 \%$ of cells in MP expressed OTR whereas only $5.32 \pm 0.06 \%$ expressed OTR in SP population. These FACS data referring to the distribution
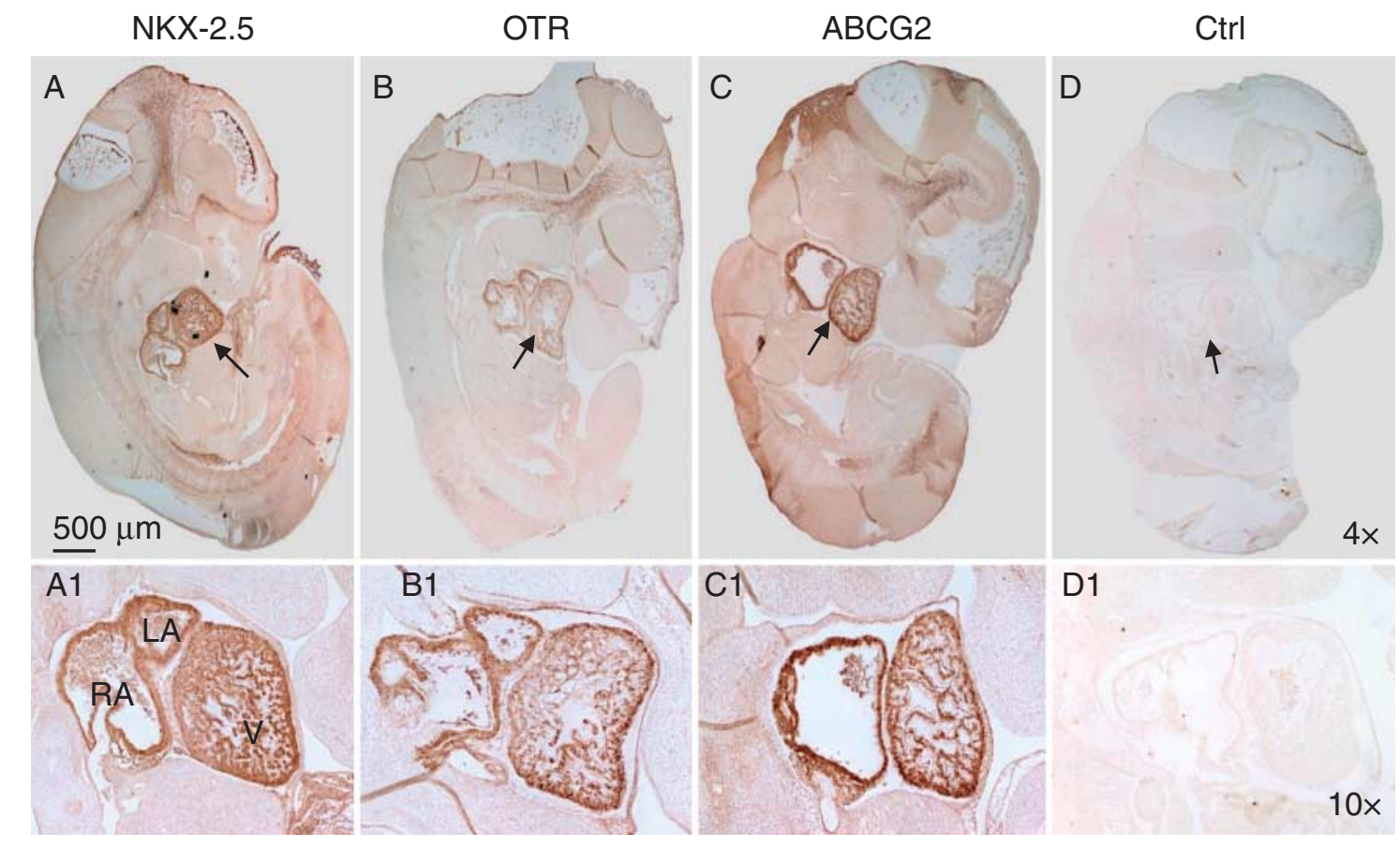

Figure 1

Immunodetection of homeobox protein NKX-2.5, oxytocin receptor (OTR), and ATP-binding cassette sub-family G member 2 (ABCG2) transporters in the heart of rat embryo (E11). The images of staining with selective antibodies in whole embryo and in the heart are presented respectively on ( $A$ and $A 1$ ) for NKX-2.5, (B and B1) for OTR, and (C and $C 1$ ) for ABCG2.
Representative control staining with isotypic antibody of whole embryo (D) and fetal heart (D1). The arrow indicates the location of the heart in the embryo. RA, right atrium; LA, left atrium; $\mathrm{V}$, ventricle. The observations were made in three embryos obtained from different litters. http://joe.endocrinology-journals.org DOI: 10.1530/JOE-13-0305
() 2014 Society for Endocrinology Printed in Great Britain 

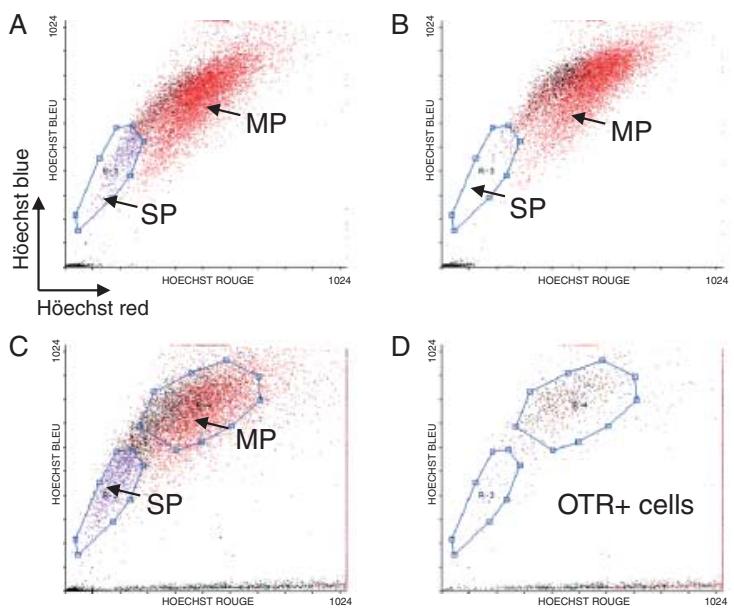

Figure 2

Isolation of side population (SP) from rat neonatal cardiac cells and their OTR expression. (A) Cardiac cells from the heart of newborn rat are stained with Höechst 33342 dye and separated into SP and main population (MP) by a dual wavelength fluorescence-activated cell sorter (FACS).

(B) Treatment with verapamil, an ATP-binding cassette transporter inhibitor, abrogated the ability of SP cells to efflux the Höechst 33342 dye.

of OTR in MP and SP population were consistent with immunofluorescence image of OTR staining of the cell culture of isolated SP (Fig. 2E) and MP (Fig. 2F) cells. Nonspecific binding of cells with goat antibody minimally influenced the results of FACS separation. As presented on the Fig. 2G, the unspecific selection of SP cells by isotype goat antibody by FACS was $0.22 \%$ as compared with $5.34 \%$ cells isolated with goat OTR-specific antibody (Fig. 2H).

The distribution of OTR + cells in endothelial, hematopoietic, and mesenchymal phenotypes of SP cells and corresponding cells of MP cells is presented in Fig. 3. The $4.7 \%$ of SP OTR + cells expressed endothelial cell antigen of PECAM1/CD31, a marker specific for developing blood vessels (Fig. 3A), 0.7\% pan-hematopoietic cell antigen CD45 (Fig. 3B), and 6.6\% $\beta-1$ integrin CD29, the marker of mesenchymal stem cells (Fig. 3C). Less frequently, OTR + cells were selected from SP cells, which represented immunotypes CD $31-(0.5 \%), \mathrm{CD} 45+(0.7 \%)$, and CD29- (0.5\%).

\section{Effect of OT treatment on the growth of rat cardiac SP cells}

Pfister et al. (2005) reported that mouse CD31-, but not CD31 + cardiac SP cells, exhibited functional cardiomyogenic differentiation in co-culture with rat CMs. Therefore, using FACS, SP CD31 - cells from cardiac rat cells were selected (Fig. 4A). The MTT cell viability assay
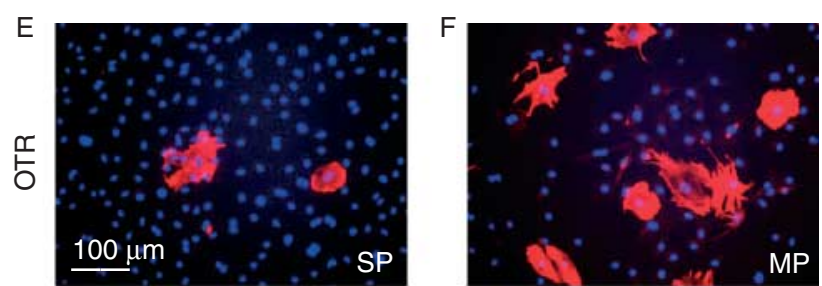

$\mathrm{G}$

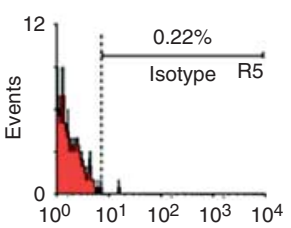

$\mathrm{H}$

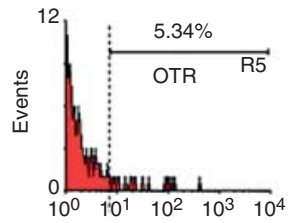

(C) Separation of cells into MP and SP. (D) Parallel flow cytometry of OTRpositive cells separated into MP and SP. (E) The identification of OTR by indirect immunofluorescence (red) in SP cell culture, and (F) MP cell culture. Nuclei stained in blue by DAPI. (G) FACS unspecific selection of SP cells with isotype goat antibody. (H) FACS isolation of SP cells using goat OTR-specific antibody. The results represent $n=3-5$.

demonstrated that the presence of $10^{-7} \mathrm{M}$ OT-GKR enhanced viability of SP CD31 - cells (Fig. 4B).

Using the hanging drop technique, SP CD31 + and SP CD31 - cells were stimulated to grow in aggregates. The SP $\mathrm{CD} 31+$ cells growing in the absence (Fig. 4C) or in the presence of OT-GKR (Fig. 4D) formed irregular cell masses. On the other hand, SP CD31- cells aggregated into spherical structures in control medium (Fig. 4E). The cell aggregates resembling embryoid bodies (EB) were enlarged in the presence of $10^{-7} \mathrm{M}$ OT-GKR (Fig. 4F). The size of aggregates, growing for 5 days in the presence of OT-GKR, averaged $194.15 \pm 18.48 \mu \mathrm{m}$ in diameter, whereas the diameter of aggregates growing only in medium measured at $89.03 \pm 1.88 \mu \mathrm{m} \quad(P<0.001$; Fig. 4I $)$. Fluorescence analysis by acridine orange and propidium iodine showed that the viability of SP CD31 - cardiac cells growing in a hanging drop is low in untreated aggregates as shown by red fluorescence, indicative of the dead cells (Fig. 4G). On the other hand, SP CD31 - cells maintained viability in the presence of the OT-GKR as indicated by green fluorescence (Fig. 4H).

\section{Development of cardiac differentiation in SP cells treated with OT-GKR}

The fraction of cardiac cells depleted from adherent cells was selected for ABCG2 antigen using magnetic beads coated with specific antibody. To examine functionality of

Published by Bioscientifica Ltd 

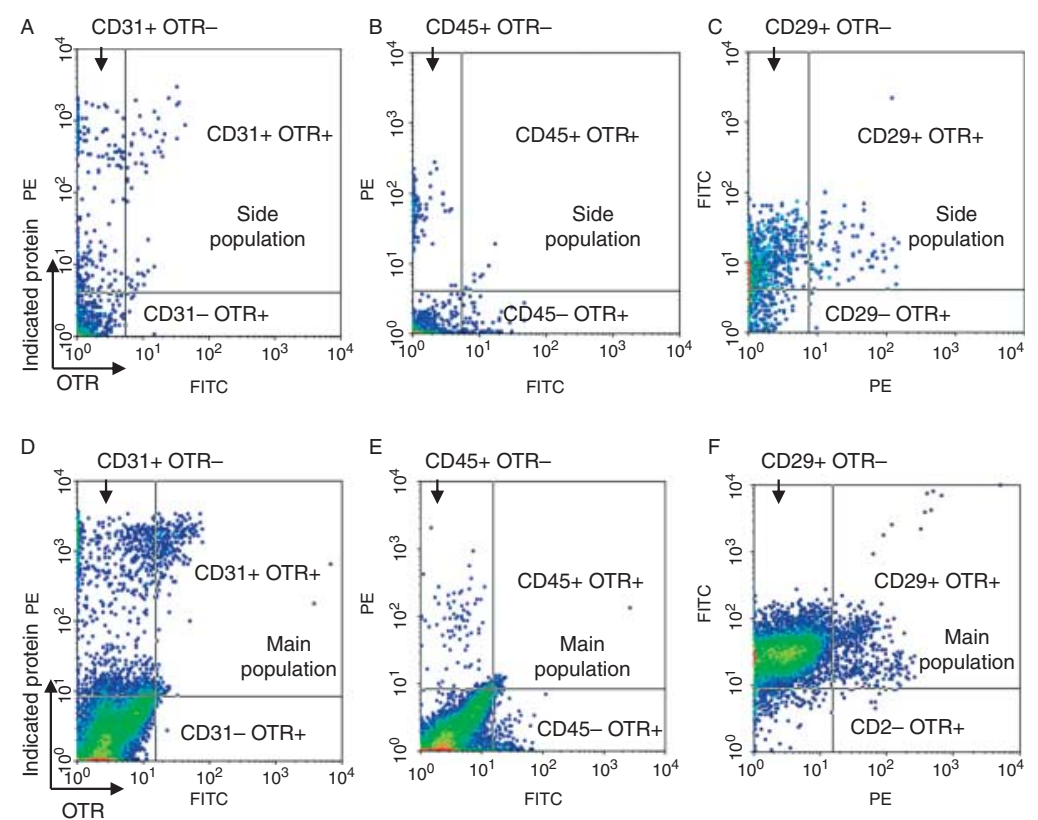

\section{Figure 3}

Distribution and expression of OTR in various subgroups of side (SP) and main populations (MP). Rat cardiac SP cells incubated with antibodies specific for OTR plus one of the other three cell surface proteins: (A) CD31 (endothelial cell antigen), (B) CD45 (hematopoietic cell antigen), and

ABCG2 transporter, the positive and negative cells for ABCG2 antigen were incubated with Höechst stain and investigated by FACS for the SP profile. As presented in the Fig. 5A, the analysis by flow cytometry revealed that $80.96 \pm 6.73 \%$ cells in ABCG 2 + fraction excluded Höechst stain whereas in ABCG2 - cell fraction only $2.5 \pm 0.94 \%$ displayed SP profile (Fig. 5B).

When the SP CD31 - cells or ABCG2+CD31 - cells were treated with OT-GKR $\left(10^{-7} \mathrm{M}\right)$ for 5 days in aggregates and plated, the beating cell colonies were observed under microscopy. As presented in Fig. 5C, the first beating of cells was detected after 11 days $(10 \% \pm 2.78$ beating efficiency), and the number of contracting cell colonies reached a maximum after 16 days $(52 \% \pm 1.21)$. SP CD31 + cells or SP CD31 - cells in absence of OT-GKR never produced contracting cell colonies spontaneously. Also, MP cells treated with OT-GKR detached from culture dishes within 1 week. A similar evolution of apparition of beating cell colonies treated with OT-GKR was observed in equivalent to ABCG2 + CD31 - cells isolated by magnetic beads (Fig. 5C). The first beating was detected after 11 days $(14 \% \pm 1.39)$ and reached maximum after 16 days (54\% \pm 4.16). As presented by phase-contrast microscopy, morphology of SP CD31 - cells growing in the absence of OT-GKR (Fig. 5D) was different than these cells induced
(C) CD29 ( $\beta$-1 integrin). The distribution of OTR in MP cells positive for (D) CD31, (E) CD45, and (F) CD29 is presented in lower panel. The side/MP expressing the indicated proteins is shown in quadrant. Similar data were obtained in two independent experiments.

by this peptide (Fig. 5E). OT-GKR induced round clusters of the cell colonies displaying synchronized contractions. The production of contracting cell colonies in SP CD31cells was associated with the expression of CM-specific antigens. Only $15 \%$ of SP CD31 - cells grown in control medium displayed staining with $\alpha$-actinin antibody which are specific for cardiac and skeletal muscles (Fig. 5G1) but not with MLC2V (Fig. 5F1) and cardiac troponin antibody (Fig. 5H1) specifically targeting CM. OT-GKR-treated SP CD31 - cells were stained clearly for all tested markers such as actinin (Fig. 5F2), MLC2V (Fig. 5G2), and cardiac troponin (Fig. 5H2). Staining of the control (Fig. 5I1) and OT-GKR-treated SP CD31- cells (Fig. 5I2) revealed reduced expression of nestin protein, a marker of pluripotent stem cells in OT-GKR differentiated cells.

\section{Development of endothelial differentiation in SP cells treated with OT-GKR}

When SP CD31 - cells were grown on Matrigel no changes in cell arrangement were found (Fig. 6A), whereas in culture supplemented with OT-GKR $\left(10^{-7} \mathrm{M}\right)$, several cells clustered together and began to form a network of tubular cells after 4 days (Fig. 6B). The endothelial phenotype of these cells was confirmed by positive staining with

Published by Bioscientifica Ltd. 

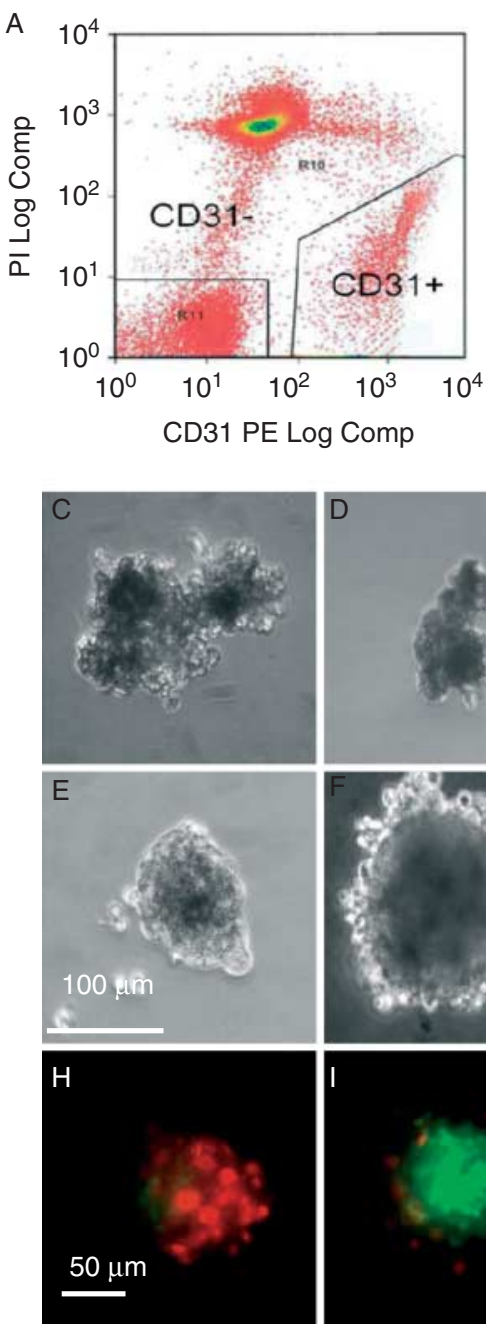

SP
B
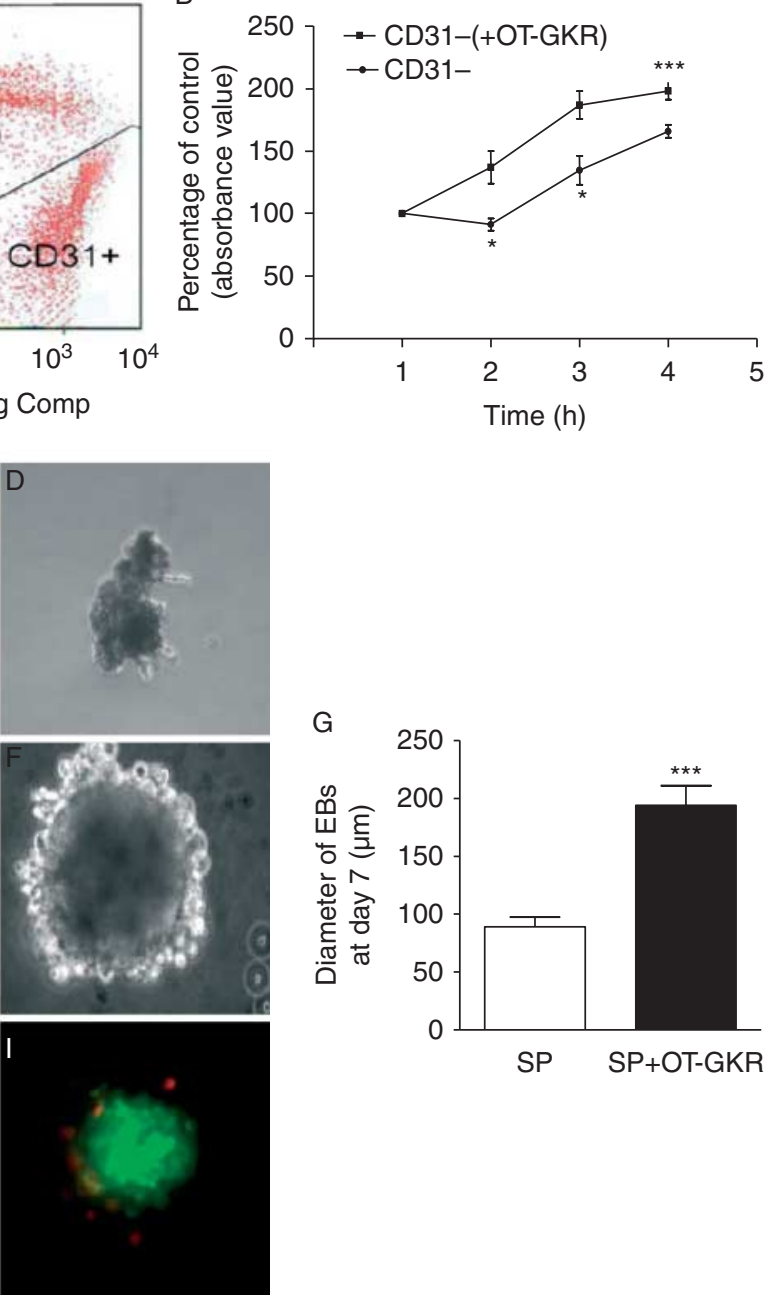

$\mathrm{SP}+\mathrm{OT}-\mathrm{GKR}$

\section{Figure 4}

Treatment with oxytocin-Gly-Lys-Arg (OT-GKR) enhances the proliferation of SP CD31 - cells and influences the shaping of cells into aggregates. (A) FACS window illustrating the separation of SP cells into CD31+ and CD31 - fractions. (B) Effect of OT-GKR $\left(10^{-7} \mathrm{M}\right)$ on the viability of SP CD31 - cells in MTT cell proliferation assay. (C, D, E, F, G, H, I) Representative images of SP cell aggregates after 7 days of incubation in hanging drop culture: (C) CD31 + cells grown in medium; (D) CD31 + cells in medium with $10^{-7} \mathrm{M}$ OT-GKR; (E) CD31 - in medium; (F) CD31 - in medium with $10^{-7} \mathrm{M}$

anti-vWF antibody (Fig. 6D). Stained cells clearly displayed the Weibel-Palade bodies, which are the storage granules of endothelial cells (Fig. 6F). In the control cultures growing in the absence of OT-GKR, no tube network or vWF-positive cells were found (Fig. 6C and E).

\section{Discussion}

In the current study, immunocytochemistry demonstrated that OTR is highly expressed in the embryonic rat heart.
OT-GKR. (G) Diameter of SP CD31 - cell aggregates growing in the presence and absence of $10^{-7} \mathrm{M}$ OT-GKR. The results were expressed as the mean \pm S.E.M. from three independent experiments $* * * P<0.001 * P<0.05$. $(\mathrm{H}$ and $\mathrm{I})$ Fluorometric cell viability assay using acridine orange and propidium iodide shows viable cells in green whereas dead cells emitted red fluorescence SP CD31 - cell aggregates, diameter developed in the absence $(\mathrm{H})$ and the presence (I) of $10^{-7} \mathrm{M}$ OT-GKR.

Using flow cytometry and immunofluorescence analysis, it has been shown that extracellular OTR is present in a small fraction of SP cells and more frequently in MP fraction of rat neonatal cardiac cells. The OTR was detected predominantly in CD29 and CD31 but less in CD45-positive SP cell populations. Although SP CD31 - cells expressed OTR in low fractions, the OT-GKR stimulation induced SP CD31 - cells to form large cell aggregates, increase in beating cell colony and endothelial formation. These data and previous findings suggest that OT-GKR can be 
A

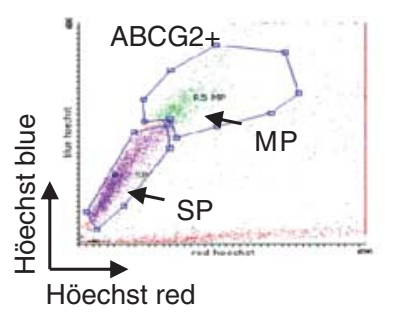

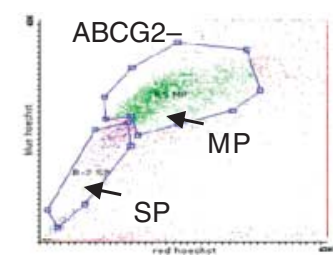

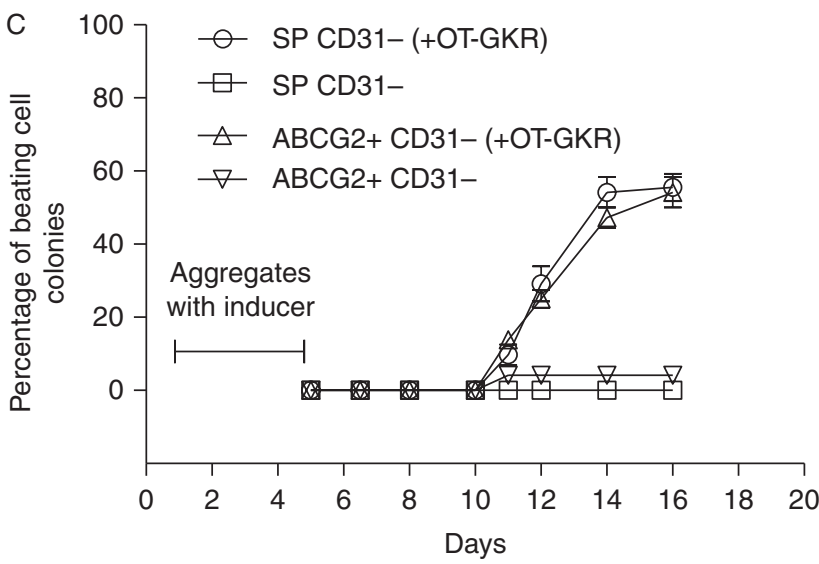

Figure 5

OT-GKR treatment of SP CD31 - cells and the equivalent, magnetic isolated $C D 31-A B C G 2$ + cells induce generation of beating colonies and $C M$ markers. (A) Identification of SP by efflux of Höechst 33342 profile in cardiomyocyte-enriched cell fraction selected by magnetic beads coated with $A B C G 2$ antibody, and (B) these cells negative for $A B C G 2$ antigen. (C) Time course appearance of beating cell colonies of SP CD31 - cells after treatment with OT-GKR and plating. The results (mean \pm s.E.M.) are from three independent differentiation experiments of cells isolated by flow cytometry or by magnetic beads. (D) Morphology of control SP CD31 - cells

involved in cardiac development. However, these results must be interpreted with care because cell behavior and binding to receptors can be unique in a living organism where many factors can influence the interaction (concentration, temperature, $\mathrm{pH}$, etc.).

Previous studies demonstrated the presence of OT and OTRs in the heart with the data supporting a potential role of OT in cardiomyogenesis. The elevated OT and OTR protein levels were observed in the developing rat heart at day 21 of gestation and postnatal days $1-4$, when CMs are at a stage of intense hyperplasia (Jankowski et al. 2004). At the end of gestation, the immunofluorescence indicated OT-GKR deposits in cells stained with the CM marker troponin C, in fetal heart sections (Danalache et al. 2010).
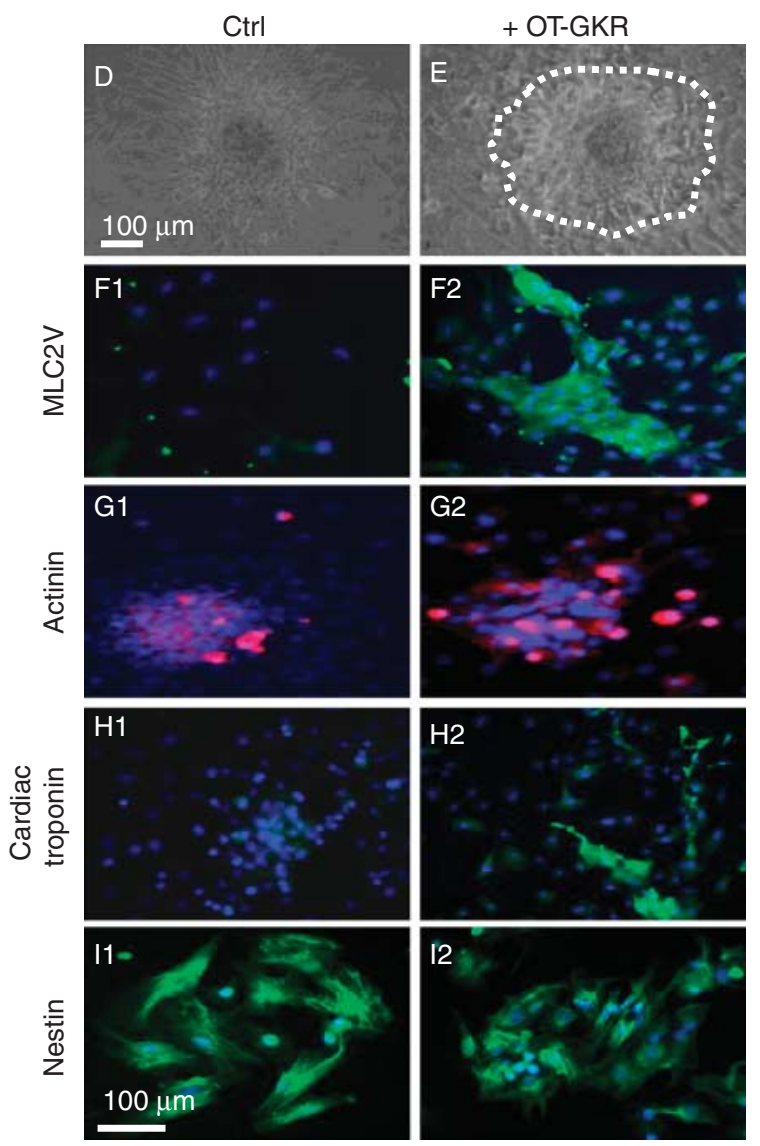

at day 14. (E) Image of SP CD31 - beating cell colony induced by OT-GKR treatment on day 14. Dotted lines encircle the contracting cell colonies. Immunofluorescence of cell differentiation markers in SP CD31 - cells of non-induced controls and in cells stimulated to differentiate with OT-GKR. $\alpha$-actinin protein for cardiac and skeletal muscles (F1 and F2), ventricular myosin light chain-2 ventricular (MLC2V) (G1 and G2), cardiac troponin T ( $\mathrm{H} 1$ and $\mathrm{H} 2$ ), and nestin (I1 and I2). For detection, appropriate secondary antibodies Alexa Fluor were used, and nuclei were counterstained in blue with DAPI. $n=3$.

The cells were also stained with anti-OTR antibody and with anti-OT antibody, which was hardly visible (Danalache et al. 2010). Between postnatal days 1 and 66, OT concentration decreased linearly in the heart and OTRs, which were elevated in postnatal $\mathrm{CM}$, declined with age to low levels in adults. Cardiac cells expressing OTR include CMs (Jankowski et al. 1998), endothelial (Jankowski et al. 2004), and neural lineages (Mukaddam-Daher et al. 2001). Interestingly, in the coronary vasculature, OTRs developed in endothelial cells at postnatal days 12 and 22 and achieved a plateau in adult rats. The decline of cardiac OT expression from infancy to adulthood of the rat and the changes in cell types expressing OTR indicate a dynamic regulation of the OT system in the heart. 

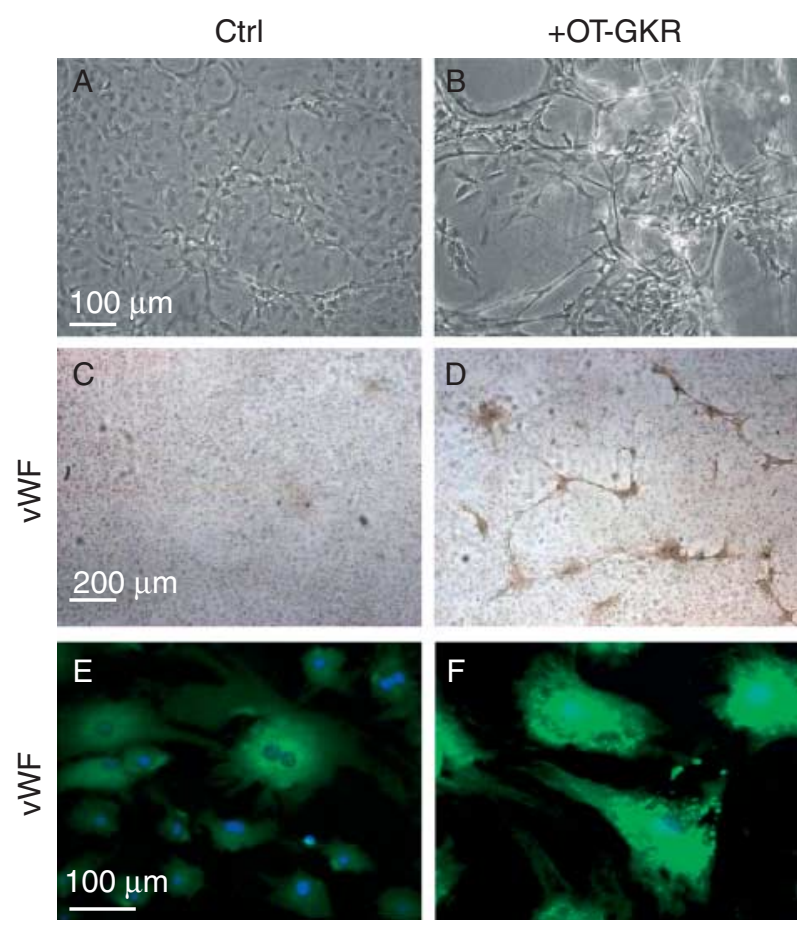

Figure 6

SP CD31 - cells grown on Matrigel in the presence of OT-GKR at day 4. The microphotographs show images of culture made in phase contrast (A and B), stained with anti-von Willebrand antibody in bright field ( $C$ and $D)$, and UV fluorescence for Alexa Fluor 488 ( $E$ and $F$ ) in presence of OT-GKR ( $B, D$ and $F$ ) and in the absence of the peptide ( $A, C$ and $E$ ). Weibel-Palade bodies (F). Ctrl, control. $n=5$.

We initiated our study of OT-GKR in cardiomyogenic differentiation because previously we have observed that OT-GKR but not OT abundantly accumulates in the fetal rodent hearts and was seen inside cells expressing the CM markers (Danalache et al. 2010). Oyama et al. (2007) revealed that rat cardiac SP cells growing in the monolayer, when treated with OT, migrated and differentiated into beating $\mathrm{CM}$, endothelial cells, and smooth muscle cells. In their study, fraction of $5 \%$ of cardiac SP cells differentiated into $\mathrm{CM}$ with fine sarcomere structures and spontaneous beating after 4 weeks. It has been previously proposed that the cardiac SP cells function as a progenitor cell population for the development, maintenance, and repair of the heart (Martin et al. 2004). Presently, using immunocytochemistry, after screening of different stage of development, we have observed in the heart of rat embryos at an early stage of development (E11), the expression of cardiac differentiation marker NKX-2.5, the accumulation of the SP cell-specific ABCG2 antigen, and OTR protein. However, FACS selection of OTR-positive cells from the newborn rat heart revealed the presence of fewer cells in the SP population than in the MP.
In order to better identify potential OT-GKR targets among the cells of cardiac SP, we analyzed the distribution of OTRs in major group of the stem cells expressing hematopoietic (CD45), mesenchymal (CD29), and endothelial (CD31) markers. Among the adult stem cells, hematopoietic stem cells are the most characterized adult stem cell population possessing cardiomyogenic potential (Leri et al. 2011). However, in our study these cells expressing the CD45 marker composed only a small fraction of SP cells, which is consistent with the other reports (Oyama et al. 2007), and its minor role in cardiac regeneration (Leri et al. 2011). Furthermore, we observed that the OTR + cells in the SP were relatively enriched in CD31-positive, CD29-positive, and CD45 negative cell subsets, which have not been reported as CM precursors. These cells are located within the vascular endothelium (Oyama et al. 2007) and can serve as a target of OTmediated angiogenesis (Cattaneo et al. 2008). On the other hand, the ABCG2-CD29-positive and CD31-negative cells are detected in the perivascular region and myocardial interstitium at the interface with CMs (Oyama et al. 2007). These cells can initiate generation of CMs when stimulated by OT (Matsuura et al. 2004). In young mice, among SP cells, the CD31 - represented only a fraction of $10 \%$, but importantly, only the Sca1-positive cardiac SP CD31cells present cardiomyogenic potential (Pfister et al. 2005). In regards to differentiation of mouse SP CD31 - , but not SP CD31 + cells into functional CMs, Liang et al. (2010) reported that CD31 - cells transplanted into an infarcted mouse heart, modestly expressed $(\sim 4 \%)$ troponin $\mathrm{T}$ and cardiac $\alpha$-actinin, the markers of CM differentiation. Interestingly, they also found that some of the transplanted SP CD31- cells expressed endothelial-specific markers including vWF. In the recent studies, Emmert et al. (2012) noted an increased number of human ABCG2+ CD31 - and ABCG2 + CD31 + cells in the peri-infarct area of the ventricle in the patients with myocardial infarction. Ex vivo $\mathrm{ABCG} 2+\mathrm{CD} 31$ - cells responded to OT treatment with increase in $\alpha$-myosin heavy chain expression (Emmert et al. 2012). Altogether, these studies indicate that stimulation of SP CD31 - cells in injured heart may have clinical implications and that effect of OT (OT-GKR) on these cells warrants further study.

Data presented in this study indicate that OT-GKR possesses the capacity of cardiac differentiation of SP cells. Using the MTT test, we show that the precursor of OT, OT-GKR, enhanced the viability of SP CD31 - cells. Our previous study on cardiomyogenesis in an embryonic stem cell line D3 demonstrated that inhibition of OTR by specific antagonist (OTA) did not reduced the number

Published by Bioscientifica Ltd. 
of OT-GKR-stimulated beating EBs to control levels. This is contrasted with the observation of Oyama et al. (2007) that OT antagonist (OTA) completely inhibits the production of CM markers in SP cells stimulated by the amidated form of OT. This indicates the presence of other pathways transducing cardiomyogenesis in response to OT-GKR treatment. Because molecular modeling revealed OT-GKR docking to active OTR sites and to V1a receptor of AVP, it appears likely that both receptors are targets of OT-GKR (Danalache et al. 2010). Indeed, it has been shown that AVP, via AVP receptor 1 (V1aR), promotes CM differentiation (Gassanov et al. 2007, Gutkowska et al. 2007). Moreover, the V1aR and AVP receptor 2 (V2R) are highly expressed and functionally active during ES cellderived cardiomyogenesis (Gassanov et al. 2007). A Ca ${ }^{2+}$. mobilization study in D3 cells demonstrated functional OT-GKR activity in embryonic stem cells (Gassanov et al. 2008). The observed sustained effect on $\mathrm{Ca}^{2+}$ may be due to the nature of OTR and V1aR. Further, data from these studies suggest that both receptors activated eNOS (Gassanov et al. 2007, Danalache et al. 2010). OT-mediated eNOS activation is signaled through a PLC/calcium/ calmodulin pathway(Leri et al. 2011); however, we have demonstrated that in CM and SP cells the phosphatidylinositol-3-kinase/AKT pathway (Florian et al. 2010) can also lead to enhancement of eNOS activity (Cattaneo et al. 2008).

The cell aggregates of these cells, when primed with OT-GKR, differentiated into beating cell colonies expressing CM markers. Staining of the control and SP CD31 - cells revealed the expression of nestin protein a marker of pluripotent stem cells (Tomita et al. 2005), which is expressed early in neuronal differentiation. The expression of nestin, which is highly expressed in undifferentiated SP cells, is lowered following the differentiation with OT-GKR. It is possible that in the presence of OT-GKR cardiac resident neural stem cells can be targeted/switched for muscle differentiation.

From the observations of Uchida et al. (2007) on the P19 cell clone 6 , we concluded that OT can more efficiently stimulate cardiomyogenesis of stem cells when they aggregate to EB rather than in monolayer culture. Indeed, the efficient differentiation of EC P19 cells depends on the prior formation of non-adhering cell aggregates, which promotes generation of mesodermal or ectodermal lineages and mimic many of the hallmarks of early embryonic development (van der Heyden \& Defize 2003). Correspondingly, we have demonstrated that rat CD31 - SP cells, but not CD31+ SP cells in response to OT-GKR treatment, self-organize to create regular three-dimensional structures in aggregates and then differentiate effectively into beating cell colonies expressing CM markers: troponin, actinin, and MLC2V. Differentiation took place in the relatively low fraction of rat cardiac SP CD31 - cells (0.5\%) being positive for OTR as immunodetected by FACS. In response to OT-GKR treatment, the $10 \%$ of aggregates of SP CD31- cells started to differentiate into beating cell colonies at day 11 after cell plating. The ability of cardiomyogenic differentiation of SP CD31 - cells by OT-GKR was linked with regular shape and high dimension of cell aggregates generated in the presence of the peptide. Aggregate size affects cells differentiation with the cavities and trabecula inside the aggregates creating a specific sponge-like environment promoting the secretion of growth factors (Bratt-Leal et al. 2009). The conditions inside aggregates such as hypoxia can stimulate OTR expression as noticed in P19 cells (Paquin et al. 2002).

The SP CD31 - cells having stronger responses to OTGKR treatment displayed very low fractions of OTR + cells. It is possible that an immunological method of OTR detection on the live cell surface is not sensitive enough to reveal all transducing receptors (Reversi et al. 2006). Other limitations include the restricted specificity and affinity of antibodies, the OTR localization within caveolin-1enriched membrane domains, OTR colocalization with lipid rafts in the cells (Cassoni et al. 2006, Reversi et al. 2006), and the movement of OTR into cell nuclei (Noiseux et al. 2012), as well as the OTR dimerization with V1aR and V2R (Devost \& Zingg 2003).

In summary, our study strongly suggests that the OTGKR treatment of SP CD31 - cells stimulates contractile cells, where the expression of cardiac markers: troponin, MLC2V, and actinin, showed a cardiac phenotype. It is also possible that during cardiac development the OT-GKR stimulates the combined actions of cardiac differentiation, glucose uptake, and the generation of capillary network. These multiple synergistic actions of OT-GKR acting on specific set of receptors make them attractive targets for therapy in heart disease. In pathological conditions, such as cardiac ischemia and diabetes, OT-GKR can be used to stimulate the production of lost cardiac cells. Moreover, it is now possible to transplant one's own stem cells after previous stimulation with OT inducers, as in the case of a heart infarct.

\section{Supplementary data}

This is linked to the online version of the paper at http://dx.doi.org/10.1530/ JOE-13-0305.

Published by Bioscientifica Ltd. 


\section{Declaration of interest}

The authors declare that there is no conflict of interest that could be perceived as prejudicing the impartiality of the research reported.

\section{Funding}

This research was supported by the Canadian Institutes of Health Research (MOP-97992, MOP-62901 to J G and M J), the Canadian Heart and Stroke Foundation (to $\mathrm{J} \mathrm{G}$ and $\mathrm{M} \mathrm{J}$ ). The funders had no role in study design, data collection and analysis, decision to publish, or preparation of the manuscript.

\section{References}

Altstein M \& Gainer H 1988 Differential biosynthesis and posttranslational processing of vasopressin and oxytocin in rat brain during embryonic and postnatal development. Journal of Neuroscience 8 3967-3977.

Anvari MA, Imani A, Faghihi M, Karimian SM, Moghimian M \& Khansari M 2012 The administration of oxytocin during early reperfusion, dosedependently protects the isolated male rat heart against ischemia/ reperfusion injury. European Journal of Pharmacology 682 137-141. (doi:10.1016/j.ejphar.2012.02.029)

Bratt-Leal AM, Carpenedo RL \& McDevitt TC 2009 Engineering the embryoid body microenvironment to direct embryonic stem cell differentiation. Biotechnology Progress 25 43-51. (doi:10.1002/btpr.139)

Cassoni P, Marrocco T, Bussolati B, Allia E, Munaron L, Sapino A \& Bussolati G 2006 Oxytocin induces proliferation and migration in immortalized human dermal microvascular endothelial cells and human breast tumor-derived endothelial cells. Molecular Cancer Research 4 351-359. (doi:10.1158/1541-7786.MCR-06-0024)

Cattaneo MG, Chini B \& Vicentini LM 2008 Oxytocin stimulates migration and invasion in human endothelial cells. British Journal of Pharmacology 153 728-736. (doi:10.1038/sj.bjp.0707609)

Danalache BA, Gutkowska J, Slusarz MJ, Berezowska I \& Jankowski M 2010 Oxytocin-Gly-Lys-Arg: a novel cardiomyogenic peptide. PLOS ONE $\mathbf{5}$ e13643. (doi:10.1371/journal.pone.0013643)

Devost D \& Zingg HH 2003 Identification of dimeric and oligomeric complexes of the human oxytocin receptor by co-immunoprecipitation and bioluminescence resonance energy transfer. Journal of Molecular Endocrinology 31 461-471. (doi:10.1677/jme.0.0310461)

Emmert MY, Emmert LS, Martens A, Ismail I, Schmidt-Richter I, Gawol A, Seifert B, Haverich A, Martin U \& Gruh I 2012 Higher frequencies of $\mathrm{BCRP}+$ cardiac resident cells in ischaemic human myocardium. European Heart Journal 34 2830-8. (doi:10.1093/eurheartj/ehs156)

Fisher DJ, Heymann MA \& Rudolph AM 1980 Myocardial oxygen and carbohydrate consumption in fetal lambs in utero and in adult sheep. American Journal of Physiology 238 H399-H405.

Florian M, Jankowski M \& Gutkowska J 2010 Oxytocin increases glucose uptake in neonatal rat cardiomyocytes. Endocrinology 151 482-491. (doi:10.1210/en.2009-0624)

Gassanov N, Jankowski M, Danalache B, Wang D, Grygorczyk R, Hoppe UC \& Gutkowska J 2007 Arginine vasopressin-mediated cardiac differentiation: insights into the role of its receptors and nitric oxide signaling. Journal of Biological Chemistry 282 11255-11265. (doi:10.1074/jbc. M610769200)

Gassanov N, Devost D, Danalache B, Noiseux N, Jankowski M, Zingg HH \& Gutkowska J 2008 Functional activity of the carboxyl-terminally extended oxytocin precursor Peptide during cardiac differentiation of embryonic stem cells. Stem Cells 26 45-54. (doi:10.1634/stemcells. 2007-0289)

Gutkowska J \& Jankowski M 2012 Oxytocin revisited: its role in cardiovascular regulation. Journal of Neuroendocrinology 24 599-608. (doi:10.1111/j.1365-2826.2011.02235.x)
Gutkowska J, Miszkurka M, Danalache B, Gassanov N, Wang D \& Jankowski M 2007 Functional arginine vasopressin system in early heart maturation. American Journal of Physiology. Heart and Circulatory Physiology 293 H2262-H2270. (doi:10.1152/ajpheart.01320.2006)

van der Heyden MA \& Defize LH 2003 Twenty one years of P19 cells: what an embryonal carcinoma cell line taught us about cardiomyocyte differentiation. Cardiovascular Research 58 292-302. (doi:10.1016/ S0008-6363(02)00771-X)

Jankowski M, Hajjar F, Kawas SA, Mukaddam-Daher S, Hoffman G, McCann SM \& Gutkowska J 1998 Rat heart: a site of oxytocin production and action. PNAS 95 14558-14563. (doi:10.1073/pnas.95. 24.14558)

Jankowski M, Danalache B, Wang D, Bhat P, Hajjar F, Marcinkiewicz M, Paquin J, McCann SM \& Gutkowska J 2004 Oxytocin in cardiac ontogeny. PNAS 101 13074-13079. (doi:10.1073/pnas.0405324101)

Jankowski M, Bissonauth V, Gao L, Gangal M, Wang D, Danalache B, Wang Y, Stoyanova E, Cloutier G, Blaise G et al. 2010 Antiinflammatory effect of oxytocin in rat myocardial infarction. Basic Research in Cardiology 105 205-218. (doi:10.1007/s00395-009-0076-5)

Kobayashi H, Yasuda S, Bao N, Iwasa M, Kawamura I, Yamada Y, Yamaki T, Sumi S, Ushikoshi H, Nishigaki K et al. 2009 Postinfarct treatment with oxytocin improves cardiac function and remodeling via activating cellsurvival signals and angiogenesis. Journal of Cardiovascular Pharmacology 54 510-519. (doi:10.1097/FJC.0b013e3181bfac02)

Leri A, Kajstura J \& Anversa P 2011 Role of cardiac stem cells in cardiac pathophysiology: a paradigm shift in human myocardial biology. Circulation Research 109 941-961. (doi:10.1161/CIRCRESAHA.111. 243154)

Liang SX, Tan TY, Gaudry L \& Chong B 2010 Differentiation and migration of Sca $1+/ C D 31$ - cardiac side population cells in a murine myocardial ischemic model. International Journal of Cardiology 138 40-49. (doi:10.1016/j.ijcard.2008.08.032)

Martin CM, Meeson AP, Robertson SM, Hawke TJ, Richardson JA, Bates S, Goetsch SC, Gallardo TD \& Garry DJ 2004 Persistent expression of the ATP-binding cassette transporter, Abcg2, identifies cardiac SP cells in the developing and adult heart. Developmental Biology 265 262-275. (doi:10.1016/j.ydbio.2003.09.028)

Matsuura K, Nagai T, Nishigaki N, Oyama T, Nishi J, Wada H, Sano M, Toko H, Akazawa H, Sato T et al. 2004 Adult cardiac Sca-1-positive cells differentiate into beating cardiomyocytes. Journal of Biological Chemistry 279 11384-11391. (doi:10.1074/jbc.M310822200)

Mitchell BF, Fang X \& Wong S 1998 Oxytocin: a paracrine hormone in the regulation of parturition? Reviews of Reproduction 3 113-122. (doi:10.1530/ror.0.0030113)

Morris M, Castro M \& Rose JC 1992 Alterations in oxytocin prohormone processing during early development in the fetal sheep. American Journal of Physiology 263 R738-R740.

Mueller-Heubach E, Morris M \& Rose JC 1995 Fetal oxytocin and its extended forms at term with and without labor. American Journal of Obstetrics and Gynecology 173 375-380 discussion 380-371. (doi:10.1016/0002-9378(95)90255-4)

Mukaddam-Daher S, Yin YL, Roy J, Gutkowska J \& Cardinal R 2001 Negative inotropic and chronotropic effects of oxytocin. Hypertension 38 292-296. (doi:10.1161/01.HYP.38.2.292)

Noiseux N, Borie M, Desnoyers A, Menaouar A, Stevens LM, Mansour S, Danalache BA, Roy DC, Jankowski M \& Gutkowska J 2012 Preconditioning of stem cells by oxytocin to improve their therapeutic potential. Endocrinology 153 5361-5372. (doi:10.1210/en.2012-1402)

Olson EN 2006 Gene regulatory networks in the evolution and development of the heart. Science $\mathbf{3 1 3}$ 1922-1927. (doi:10.1126/science. 1132292)

Ondrejcakova M, Ravingerova T, Bakos J, Pancza D \& Jezova D 2009 Oxytocin exerts protective effects on in vitro myocardial injury induced by ischemia and reperfusion. Canadian Journal of Physiology and Pharmacology 87 137-142. (doi:10.1139/Y08-108) 
Oyama T, Nagai T, Wada H, Naito AT, Matsuura K, Iwanaga K, Takahashi T, Goto M, Mikami Y, Yasuda N et al. 2007 Cardiac side population cells have a potential to migrate and differentiate into cardiomyocytes in vitro and in vivo. Journal of Cell Biology 176 329-341. (doi:10.1083/jcb. 200603014)

Paquin J, Danalache BA, Jankowski M, McCann SM \& Gutkowska J 2002 Oxytocin induces differentiation of P19 embryonic stem cells to cardiomyocytes. PNAS 99 9550-9555. (doi:10.1073/pnas. 152302499)

Pfister O, Mouquet F, Jain M, Summer R, Helmes M, Fine A, Colucci WS \& Liao R 2005 CD31 - but Not CD31 + cardiac side population cells exhibit functional cardiomyogenic differentiation. Circulation Research 97 52-61. (doi:10.1161/01.RES.0000173297.53793.fa)

Reversi A, Rimoldi V, Brambillasca S \& Chini B 2006 Effects of cholesterol manipulation on the signaling of the human oxytocin receptor. American Journal of Physiology. Regulatory, Integrative and Comparative Physiology 291 R861-R869. (doi:10.1152/ajpregu.00333.2006)
Schindelin J, Arganda-Carreras I, Frise E, Kaynig V, Longair M, Pietzsch T, Preibisch S, Rueden C, Saalfeld S, Schmid B et al. 2012 Fiji: an open-source platform for biological-image analysis. Nature Methods 9 676-682. (doi:10.1038/nmeth.2019)

Tomanek RJ, Haung L, Suvarna PR, O'Brien LC, Ratajska A \& Sandra A 1996 Coronary vascularization during development in the rat and its relationship to basic fibroblast growth factor. Cardiovascular Research $\mathbf{3 1}$ E116-E126.

Tomita Y, Matsumura K, Wakamatsu Y, Matsuzaki Y, Shibuya I, Kawaguchi H, Ieda M, Kanakubo S, Shimazaki T, Ogawa S et al. 2005 Cardiac neural crest cells contribute to the dormant multipotent stem cell in the mammalian heart. Journal of Cell Biology 170 1135-1146. (doi:10.1083/jcb.200504061)

Uchida S, Fuke S \& Tsukahara T 2007 Upregulations of Gata4 and oxytocin receptor are important in cardiomyocyte differentiation processes of P19CL6 cells. Journal of Cellular Biochemistry 100 629-641. (doi:10.1002/jcb.21094)

Received in final form 2 December 2013

Accepted 6 January 2014

Accepted Preprint published online 8 January 2014
C 2014 Society for Endocrinology Printed in Great Britain
Published by Bioscientifica Ltd. 